\title{
Asymptotic Uniqueness for a Biharmonic Equation with Nearly Critical Growth on Symmetric Convex Domains
}

\author{
By \\ Tomohiko SATo and Futoshi TAKahashi \\ (Gakushuin University and Osaka City University, Japan)
}

\begin{abstract}
We consider a biharmonic equation with the nearly critical Sobolev exponent under the Navier boundary condition on a smooth bounded, strictly convex domain of dimension $N \geq 5$, which is symmetric with respect to the coordinate hyperplanes.

We prove that the number of positive solutions of the above problem is exactly one when the nonlinear exponent is subcritical and sufficiently near to the critical exponent. Furthermore, this unique solution is nondegenerate in the sense that the associated linearized problem admits only the trivial solution.

Key Words and Phrases. Biharmonic equation, Critical exponent.

2000 Mathematics Subject Classification Numbers. 35B33, 35J35, 35 J60.
\end{abstract}

\section{Introduction}

We consider the problem $\left(P_{\varepsilon}\right)$ with the Navier boundary condition:

$$
\left(P_{\varepsilon}\right) \begin{cases}\Delta^{2} u=c_{0} u^{p_{\varepsilon}} & \text { in } \Omega, \\ u>0 & \text { in } \Omega, \\ u=\Delta u=0 & \text { on } \partial \Omega\end{cases}
$$

where $\Omega \subset \boldsymbol{R}^{N}(N \geq 5)$ be a smooth bounded domain, $c_{0}=(N-4)(N-2)$. $N(N+2), \varepsilon>0$ is a small positive parameter, $p_{\varepsilon}=p-\varepsilon$ and $p=(N+4) /$ $(N-4)$ is the critical Sobolev exponent from the view point of the Sobolev embedding $H^{2} \cap H_{0}^{1}(\Omega) \hookrightarrow L^{p+1}(\Omega)$. The existence of at least one solution is easy to obtain for $\varepsilon>0$ small. In this paper, we prove a uniqueness and a qualitative property of solution for the problem $\left(P_{\varepsilon}\right)$.

We impose some geometric assumptions on the domain.

(H1) $\Omega$ is symmetric with respect to the hyperplane $\left\{x_{i}=0\right\}$, $(i=1, \ldots, N)$.

(H2) $\Omega$ is strictly convex.

Note that $\left(P_{\varepsilon}\right)$ is equivalent to the cooperative system 


$$
\begin{cases}-\Delta u=\bar{u} & \text { in } \Omega, \\ -\Delta \bar{u}=c_{0} u^{p_{\varepsilon}} & \text { in } \Omega, \\ u>0, \bar{u}>0 & \text { in } \Omega, \\ u=\bar{u}=0 & \text { on } \partial \Omega .\end{cases}
$$

Therefore under the assumptions (H1) and

(H2') $\Omega$ is convex in the $x_{i}$-direction, $(i=1, \ldots, N)$, any solution $u_{\varepsilon}$ to $\left(P_{\varepsilon}\right)$ is symmetric with respect to the hyperplane $\left\{x_{i}=0\right\}$ :

$$
u_{\varepsilon}\left(x_{1}, \ldots, x_{i}, \ldots, x_{N}\right)=u_{\varepsilon}\left(x_{1}, \ldots,-x_{i}, \ldots, x_{N}\right), \quad(i=1, \ldots, N),
$$

and monotone with respect to the positive $x_{i}$-direction:

$$
\frac{\partial u}{\partial x_{i}}<0 \quad \text { for } x_{i}>0,(i=1, \ldots, N),
$$

see [16] Lemma 4.3. From these and since $\left(P_{0}\right)$ has no solution, we easily see

$$
\left\|u_{\varepsilon}\right\|_{L^{\infty}(\Omega)}=u_{\varepsilon}(0) \rightarrow+\infty \quad \text { as } \varepsilon \rightarrow 0 .
$$

In this note, we prove

Theorem 1 (Asymptotic uniqueness). Assume $\Omega \subset \boldsymbol{R}^{N}, N \geq 5$ satisfies (H1) and $(\mathrm{H} 2)$. Let $u_{\varepsilon}$ and $v_{\varepsilon}$ be two solutions to $\left(P_{\varepsilon}\right)$. Then there exists $\varepsilon_{0}>0$ such that for any $\varepsilon \in\left(0, \varepsilon_{0}\right)$, we have $u_{\varepsilon} \equiv v_{\varepsilon}$ on $\Omega$.

Theorem 2. The unique solution $u_{\varepsilon}$ to $\left(P_{\varepsilon}\right)$ in Theorem 1 is nondegenerate in the sense that $\lambda=0$ is not an eigenvalue for the linearized eigenvalue problem

$$
\begin{cases}\Delta^{2} w=c_{0} p_{\varepsilon} u_{\varepsilon}^{p_{\varepsilon}-1} w+\lambda w & \text { in } \Omega \\ w=\Delta w=0 & \text { on } \partial \Omega\end{cases}
$$

As far as we know, the uniqueness of solutions to the subcritical problem

$$
\begin{cases}\Delta^{2} u=u^{p} & \text { in } \Omega, 1<p<(N+4) /(N-4), \\ u>0 & \text { in } \Omega \\ u=\Delta u=0 & \text { on } \partial \Omega\end{cases}
$$

where $\Omega$ satisfies (H1) and (H2) (or $\left(\mathrm{H} 2^{\prime}\right)$ ), seems widely open, except for the case $\Omega$ is a ball. We note that the blow up phenomenon does induce the uniqueness result in Theorem 1.

Our argument goes along the line of Grossi [11]; see also [3]. Grossi obtained the same uniqueness and the nondegeneracy results for the problem

$$
\begin{cases}-\Delta u=N(N-2) u^{p_{\varepsilon}} & \text { in } \Omega \subset \boldsymbol{R}^{N}, N \geq 3, \\ u>0 & \text { in } \Omega, \\ u=0 & \text { on } \partial \Omega\end{cases}
$$

under the assumptions $(\mathrm{H} 1)$ and $\left(\mathrm{H} 2^{\prime}\right)$. 
In the proof, Grossi used a fine blow up analysis by YanYan Li [18] to show that the results of Han [12] hold true for general solutions of the above problem, under $(\mathrm{H} 1)$ and $\left(\mathrm{H} 2^{\prime}\right)$. In the Laplacian case, the uniform supremum estimate near the boundary for general solutions is obtained by the method of moving planes of Gidas, $\mathrm{Ni}$ and Nirenberg [10], and the additional use of the Kelvin transformation if the domain is not strictly convex.

The method of moving planes also assures that the uniform boundedness near the boundary for general solutions of $\left(P_{\varepsilon}\right)$ if the domain is strictly convex. However, in our biharmonic case, the Kelvin transformation does not work well because the Navier boundary condition is not preserved under the transformation; see [5]. This is the reason why we assume $(\mathrm{H} 2)$.

Once we confirm that a blow up point is isolated and not on the boundary, then we can employ the local blow up analysis and the theory of isolated simple blow up points, recently obtained by Djadli, Malchiodi and Ahmedou [7] for biharmonic equations. See also Felli [9]. Their works assure that the origin is an isolated simple blow up point for any solution sequence of $\left(P_{\varepsilon}\right)$, and the results of Chou and Geng [5], known to be valid for solutions minimizing the Sobolev quotient, hold true also for general solutions under (H1) and (H2).

In the proof of Theorem 2, we extend a lemma of Damascelli, Grossi and Pacella [6] to a polyharmonic problem. We hope this is itself interesting, see Lemma 13.

\section{Preliminaries}

In this section, we collect some useful facts in the sequel. Let $G=G(x, y)$ denote the Green function of $\Delta^{2}$ under the Navier boundary condition:

$$
\begin{cases}\Delta^{2} G(\cdot, y)=\delta_{y} & \text { in } \Omega, \\ G(\cdot, y)=\Delta G(\cdot, y)=0 & \text { on } \partial \Omega .\end{cases}
$$

The Robin function is defined as

$$
R(x)=\lim _{y \rightarrow x}[\Gamma(x, y)-G(x, y)]
$$

where

$$
\Gamma(x, y)= \begin{cases}\frac{1}{(N-4)(N-2) \sigma_{N}}|x-y|^{4-N}, & N \geq 5, \\ \frac{1}{\sigma_{4}} \log |x-y|^{-1}, & N=4\end{cases}
$$

and $\sigma_{N}$ is the volume of the $(N-1)$ dimensional unit sphere in $\boldsymbol{R}^{N}$. We see that $R>0$ on $\Omega$ and $R(x) \rightarrow+\infty$ as $x$ tends to the boundary of $\Omega$. 
Lemma 3 (Pohozaev identity for the Green function). The identity

$$
\int_{\partial \Omega}((x-y) \cdot \nabla G) \frac{\partial}{\partial v}(-\Delta G) d s_{x}=(N-4) R(y)
$$

holds true for any $y \in \Omega$.

Proof. See [5]. Note that there is a mistake in the claimed formula in [5].

Next lemma concerns a classical elliptic regularity for a solution to the biharmonic equation, recently obtained by Caristi and Mitidieri ([1] Theorem 4.9).

Lemma 4. Let $u \in H_{\text {loc }}^{2}(\Omega)$ be a weak solution of

$$
\Delta^{2} u=a(x) u \quad \text { in } \Omega
$$

where $a \in L^{\alpha}(\Omega)$ with $\alpha>N / 4$. Then for any $q \in(0,+\infty)$, there exist $C=$ $C(q)>0$ and $R>0$ such that for any $0<r<R$ and $y \in \boldsymbol{R}^{N}$, we have

$$
\sup _{B(y, r) \cap \Omega}|u| \leq C\left[\frac{1}{r^{N}} \int_{B(y, 2 r) \cap \Omega}|u|^{q+1} d x\right]^{1 /(q+1)} .
$$

Next lemma claims that the origin is an isolated blow up point for any solution sequence $u_{\varepsilon}$ of $\left(P_{\varepsilon}\right)$. Proof will be done by a standard blow up analysis just as in [11] Lemma A.1., because we know $u_{\varepsilon}$ is uniformly bounded in sup-norm near the boundary thanks to our assumption (H2), see [5] p. 925.

Lemma 5. Assume $(\mathrm{H} 1)$ and $(\mathrm{H} 2)$. Let $u_{\varepsilon}$ be any solution to $\left(P_{\varepsilon}\right)$. Then there exists $C>0$ independent of $\varepsilon$ such that

$$
|x|^{4 /\left(p_{\varepsilon}-1\right)} u_{\varepsilon}(x) \leq C
$$

for any $x \in \Omega$.

Under more general situation, an isolated blow up point has to be an isolated simple blow up point: see [7] Proposition 2.19 and [9]. We refer [7], [9] to the definitions of isolated, or isolated simple blow up point for our biharmonic case. Then by using the estimates for isolated simple blow up points ([7] Lemma 2.11 and Lemma 2.17), we have the followings:

Lemma 6. Assume $(\mathrm{H} 1)$ and $(\mathrm{H} 2)$. Let $u_{\varepsilon}$ be any solution to $\left(P_{\varepsilon}\right)$. Then we have

$$
\lim _{\varepsilon \rightarrow 0}\left\|u_{\varepsilon}\right\|_{L^{\infty}(\Omega)}^{\varepsilon}=1
$$

and 


$$
\frac{\int_{\Omega}\left|\Delta u_{\varepsilon}\right|^{2} d x}{\left(\int_{\Omega}\left|u_{\varepsilon}\right|^{p_{\varepsilon}+1}\right)^{2 /\left(p_{\varepsilon}+1\right)}} \rightarrow S_{N}
$$

where $S_{N}$ is the best Sobolev constant of the embedding $H^{2} \cap H_{0}^{1}(\Omega) \hookrightarrow L^{p+1}(\Omega)$.

Next theorem is the main result of Chou and Geng [5].

Theorem 7 ([5]). Assume $\Omega \subset \boldsymbol{R}^{N}, N \geq 5$ is strictly convex. Let $u_{\varepsilon}$ be a solution to $\left(P_{\varepsilon}\right)$ satisfying (2.2). Let $x_{\varepsilon} \in \Omega$ be a point such that $u_{\varepsilon}\left(x_{\varepsilon}\right)=$ $\left\|u_{\varepsilon}\right\|_{L^{\infty}(\Omega)}$. Then after passing to a subsequence, we have

(1) $\lim _{\varepsilon \rightarrow 0} x_{\varepsilon}=x_{0}$ for some interior point $x_{0} \in \Omega$.

(2) For any open neighborhood $\omega$ of $\partial \Omega$ not containing $x_{0}$, there holds

$$
\left\|u_{\varepsilon}\right\|_{L^{\infty}(\Omega)} u_{\varepsilon} \rightarrow 2(N-4)(N-2) \sigma_{N} G\left(\cdot, x_{0}\right) \quad \text { as } \varepsilon \rightarrow 0
$$

in $C^{3, \alpha}(\omega)$ for some $\alpha \in(0,1)$.

(3) There exists a constant $C>0$ independent of $\varepsilon$ and solution $u_{\varepsilon}$ such that

$$
u_{\varepsilon}(x) \leq C \frac{\left\|u_{\varepsilon}\right\|_{L^{\infty}(\Omega)}}{\left(1+\left\|u_{\varepsilon}\right\|_{L^{\infty}(\Omega)}^{4 /(N-4)}\left|x-x_{\varepsilon}\right|^{2}\right)^{(N-4) / 2}}
$$

holds for any $x \in \Omega$.

(4)

$$
\lim _{\varepsilon \rightarrow 0} \varepsilon\left\|u_{\varepsilon}\right\|_{L^{\infty}(\Omega)}^{2}=\frac{16 \sigma_{N}^{2} \Gamma(N) c_{0}}{\pi^{N / 2} N(N-4)(N+2)^{2} \Gamma(N / 2)} R\left(x_{0}\right) .
$$

By Lemma 6 (2.2), we see that the results of Theorem 7 hold for any solution sequence $u_{\varepsilon}$ to $\left(P_{\varepsilon}\right)$ with $x_{\varepsilon}=x_{0}=0$ under $(\mathrm{H} 1)$ and $(\mathrm{H} 2)$.

In what follows, we use a symbol $\|\cdot\|$ to denote the $L^{\infty}$ norm of functions. Now, let us consider the scaled function

$$
\tilde{u}_{\varepsilon}(y):=\frac{1}{\left\|u_{\varepsilon}\right\|} u_{\varepsilon}\left(\frac{y}{\left\|u_{\varepsilon}\right\|^{\left(p_{\varepsilon}-1\right) / 4}}\right), \quad y \in \Omega_{\varepsilon}:=\left\|u_{\varepsilon}\right\|^{\left(p_{\varepsilon}-1\right) / 4} \Omega .
$$

We see $0<\tilde{u}_{\varepsilon} \leq 1, \tilde{u}_{\varepsilon}(0)=1$, and $\tilde{u}_{\varepsilon}$ satisfies

$$
\begin{cases}\Delta^{2} \tilde{u}_{\varepsilon}=c_{0} \tilde{u}_{\varepsilon}^{p_{\varepsilon}} & \text { in } \Omega_{\varepsilon} \\ \tilde{u}_{\varepsilon}=\Delta \tilde{u}_{\varepsilon}=0 & \text { on } \partial \Omega_{\varepsilon}\end{cases}
$$

Since $\left\|u_{\varepsilon}\right\| \rightarrow \infty$ as $\varepsilon \rightarrow 0$, we see $\Omega_{\varepsilon} \rightarrow \boldsymbol{R}^{N}$ and by standard elliptic estimates, we have a subsequence denoted also by $\tilde{u}_{\varepsilon}$ that

$$
\tilde{u}_{\varepsilon} \rightarrow U \quad \text { compact uniformly in } \boldsymbol{R}^{N}
$$

as $\varepsilon \rightarrow 0$ for some function $U$. Passing to the limit, we obtain that $U$ is a solution of 


$$
\left\{\begin{array}{l}
\Delta^{2} U=c_{0} U^{p} \quad \text { in } \boldsymbol{R}^{N} \\
0<U \leq 1, U(0)=1, \\
\lim _{|y| \rightarrow \infty} U(y)=0 .
\end{array}\right.
$$

Thus according to the uniqueness theorem by Chang Shou Lin [4], we obtain

$$
U(y)=\left(\frac{1}{1+|y|^{2}}\right)^{(N-4) / 2} .
$$

\section{A uniqueness result}

In this section, we will prove Theorem 1. Assume the contrary that there exist solutions $u_{\varepsilon}$ and $v_{\varepsilon}$ to $\left(P_{\varepsilon}\right), u_{\varepsilon} \not \equiv v_{\varepsilon}$ for some $\{\varepsilon\} \downarrow 0$. Consider the function

$$
w_{\varepsilon}(y)=\frac{1}{\left\|u_{\varepsilon}-v_{\varepsilon}\right\|}\left(u_{\varepsilon}\left(\frac{y}{\left\|u_{\varepsilon}\right\|^{\left(p_{\varepsilon}-1\right) / 4}}\right)-v_{\varepsilon}\left(\frac{y}{\left\|u_{\varepsilon}\right\|^{\left(p_{\varepsilon}-1\right) / 4}}\right)\right)
$$

for $y \in \Omega_{\varepsilon}=\left\|u_{\varepsilon}\right\|^{\left(p_{\varepsilon}-1\right) / 4} \Omega$. It is easy to check that $w_{\varepsilon}$ solves

$$
\begin{cases}\Delta^{2} w_{\varepsilon}=c_{\varepsilon}(y) w_{\varepsilon} & \text { in } \Omega_{\varepsilon} \\ w_{\varepsilon}=\Delta w_{\varepsilon}=0 & \text { on } \partial \Omega_{\varepsilon} \\ \left\|w_{\varepsilon}\right\|=1 & \end{cases}
$$

where

$$
c_{\varepsilon}(y)=c_{0} p_{\varepsilon} \int_{0}^{1}\left[t \tilde{u}_{\varepsilon}(y)+(1-t) \tilde{v}_{\varepsilon}(y)\right]^{p_{\varepsilon}-1} d t
$$

here we set

$$
\tilde{u}_{\varepsilon}(y)=\frac{1}{\left\|u_{\varepsilon}\right\|} u_{\varepsilon}\left(\frac{y}{\left\|u_{\varepsilon}\right\|^{\left(p_{\varepsilon}-1\right) / 4}}\right) \quad \text { and } \quad \tilde{v}_{\varepsilon}(y)=\frac{1}{\left\|u_{\varepsilon}\right\|} v_{\varepsilon}\left(\frac{y}{\left\|u_{\varepsilon}\right\|^{\left(p_{\varepsilon}-1\right) / 4}}\right)
$$

for $y \in \Omega_{\varepsilon}$.

By Theorem 7 (2.5), we see $\lim _{\varepsilon \rightarrow 0}\left\|u_{\varepsilon}\right\|=\lim _{\varepsilon \rightarrow 0}\left\|v_{\varepsilon}\right\|=+\infty$ and

$$
\lim _{\varepsilon \rightarrow 0} \frac{\left\|u_{\varepsilon}\right\|}{\left\|v_{\varepsilon}\right\|}=1
$$

By this, we have $\left\|\tilde{v}_{\varepsilon}\right\|=\tilde{v}_{\varepsilon}(0)=v_{\varepsilon}(0) /\left\|u_{\varepsilon}\right\| \rightarrow 1$, so as in (2.6), we see

$$
\tilde{u}_{\varepsilon} \rightarrow U \text { and } \quad \tilde{v}_{\varepsilon} \rightarrow U \quad \text { uniformly on compact sets of } \boldsymbol{R}^{N}
$$

where $U$ is as in (2.7). Thus 


$$
c_{\varepsilon} \rightarrow c_{0} p \int_{0}^{1}[t U+(1-t) U]^{p-1} d t=c_{0} p U^{p-1}
$$

uniformly on compact sets of $\boldsymbol{R}^{N}$. Since $\left\|w_{\varepsilon}\right\|=1$, standard elliptic regularity allows us to pass to the limit in the equation (3.1). Then we get

$$
w_{\varepsilon} \rightarrow w \quad \text { uniformly on compact sets of } \boldsymbol{R}^{N}
$$

for some function $w$, and the limit function $w$ satisfies

$$
\Delta^{2} w=c_{0} p U^{p-1} w \quad \text { in } \boldsymbol{R}^{N}, \quad\|w\| \leq 1 .
$$

Since $w_{\varepsilon}$ is symmetric with respect to the hyperplanes $\left\{x_{i}=0\right\}(i=1, \ldots, N)$, we see by (3.4) that $w$ is a symmetric function.

Furthermore, arguing as in [11], we check that

$$
\int_{\Omega_{\varepsilon}}\left|\Delta w_{\varepsilon}\right|^{2} d y \leq C
$$

where $C$ is independent of $\varepsilon$. Thus by Fatou's lemma, we also have

$$
\int_{\boldsymbol{R}^{N}}|\Delta w|^{2} d y \leq C
$$

Now, we recall the classification theorem by Bartsch, Weth and Willem ([2]).

Lemma 8. Let $w$ be a solution to (3.5) with the property (3.7). Then there exist $a_{j}(j=1,2, \ldots, N), b \in \boldsymbol{R}$ such that $w$ can be written as

$$
w=\sum_{j=1}^{N} a_{j} \frac{y_{j}}{\left(1+|y|^{2}\right)^{(N-2) / 2}}+b \frac{1-|y|^{2}}{\left(1+|y|^{2}\right)^{(N-2) / 2}} .
$$

In the following, we divide the proof into several steps.

Step $1 . \quad a_{j}=0, j=1, \ldots, N$.

This is a simple consequence of the fact that $w$ is a symmetric function with respect to the hyperplanes $\left\{x_{j}=0\right\}, j=1, \ldots, N$.

Step 2. $b=0$.

By step 1, we have

$$
w=b \frac{1-|y|^{2}}{\left(1+|y|^{2}\right)^{(N-2) / 2}} .
$$

Now, we need the following lemma: In the proof, we argue as in [11] Lemma A.5 with the crucial use of Lemma 4. 
Lemma 9. Let $w_{\varepsilon}$ be a solution of (3.1). Then we have the estimate

$$
\left|w_{\varepsilon}(y)\right| \leq C \frac{1}{|y|^{N-4}} \quad \text { for } y \in \Omega_{\varepsilon} \cap\{|y| \geq \delta\}
$$

for some $C>0$ and $\delta>0$.

Proof. Consider the Kelvin transformation of $w_{\varepsilon}$ :

$$
w_{\varepsilon}^{*}(z)=|z|^{4-N} w_{\varepsilon}\left(\frac{z}{|z|^{2}}\right), \quad z \in \Omega_{\varepsilon}^{*}:=\left\{\frac{y}{|y|^{2}}: y \in \Omega_{\varepsilon}\right\} .
$$

To prove (3.8), it will be enough to show that $\left|w_{\varepsilon}^{*}\right|$ is bounded in $B(0, R) \cap \Omega_{\varepsilon}^{*}$ for some $R>0$. Direct calculation shows that

$$
\begin{aligned}
& \Delta_{z}^{2} w_{\varepsilon}^{*}(z)=|z|^{-4-N} \Delta_{y}^{2} w_{\varepsilon}(y), \quad z \in \Omega_{\varepsilon}^{*}, \\
& \Delta_{z} w_{\varepsilon}^{*}(z)=4|z|^{-2}(z \cdot v) \frac{\partial w_{\varepsilon}^{*}}{\partial v}, \quad z \in \partial \Omega_{\varepsilon}^{*}, \\
& \int_{\Omega_{\varepsilon}^{*}}\left|w_{\varepsilon}^{*}\right|^{p+1} d z=\int_{\Omega_{\varepsilon}}\left|w_{\varepsilon}\right|^{p+1} d y .
\end{aligned}
$$

Thus, $w_{\varepsilon}^{*}$ satisfies the equation

$$
\begin{cases}\Delta^{2} w_{\varepsilon}^{*}=|z|^{-8} c_{\varepsilon}\left(\frac{z}{|z|^{2}}\right) w_{\varepsilon}^{*} & \text { in } \Omega_{\varepsilon}^{*}, \\ w_{\varepsilon}^{*}=0 & \text { on } \partial \Omega_{\varepsilon}^{*}, \\ \Delta w_{\varepsilon}^{*}=4|z|^{-2}(z \cdot v) \frac{\partial w_{\varepsilon}^{*}}{\partial v}, & \text { on } \partial \Omega_{\varepsilon}^{*} .\end{cases}
$$

Now, we claim that there exists a constant $C>0$ such that

$$
\left\|a_{\varepsilon}\right\|_{L^{\infty}\left(\Omega_{\varepsilon}^{*}\right)} \leq C
$$

where

$$
a_{\varepsilon}(z):=|z|^{-8} c_{\varepsilon}\left(\frac{z}{|z|^{2}}\right) .
$$

Indeed, since $\Omega_{\varepsilon} \subset B\left(0, \gamma\left\|u_{\varepsilon}\right\|^{\left(p_{\varepsilon}-1\right) / 4}\right)$ for some $\gamma>0$, we see that $\Omega_{\varepsilon}^{*} \subset$ $\boldsymbol{R}^{N} \backslash B\left(0,1 /\left(\gamma\left\|u_{\varepsilon}\right\|^{\left(p_{\varepsilon}-1\right) / 4}\right)\right)$. By (2.4), we know that

$$
\tilde{u}_{\varepsilon}(y) \leq C U(y) \quad \text { and } \quad \tilde{v}_{\varepsilon} \leq C U(y),
$$

thus

$$
\left|c_{\varepsilon}(y)\right| \leq C U^{p_{\varepsilon}-1}(y) \quad \text { for } y \in \Omega_{\varepsilon} .
$$


Therefore, we have

$$
\begin{aligned}
|z|^{-8} c_{\varepsilon}\left(\frac{z}{|z|^{2}}\right) & \leq C|z|^{-8}\left(\frac{|z|^{2}}{1+|z|^{2}}\right)^{((N-4) / 2)\left(p_{\varepsilon}-1\right)} \\
& =C|z|^{-8+(N-4)\left(p_{\varepsilon}-1\right)} \frac{1}{\left(1+|z|^{2}\right)^{4-\varepsilon((N-4) / 2)}} \\
& \leq C|z|^{-8+(N-4)\left(p_{\varepsilon}-1\right)}=C|z|^{-\varepsilon(N-4)}
\end{aligned}
$$

Since $|z| \geq 1 /\left(\gamma\left\|u_{\varepsilon}\right\|^{\left(p_{\varepsilon}-1\right) / 4}\right)$ for $z \in \Omega_{\varepsilon}^{*}$, we have

$$
|z|^{-\varepsilon(N-4)} \leq \gamma^{\varepsilon(N-4)}\left\|u_{\varepsilon}\right\|^{\varepsilon(N-4)\left(p_{\varepsilon}-1\right) / 4} \rightarrow 1
$$

as $\varepsilon \rightarrow 0$. Here we have used (2.1). From these, we confirm that the claim (3.9).

Now, for any $R>0$, we have

$$
\begin{aligned}
\int_{\Omega_{\varepsilon}^{*} \cap B(0,2 R)}\left|w_{\varepsilon}^{*}\right|^{p+1} d z & \leq \int_{\Omega_{\varepsilon}^{*}}\left|w_{\varepsilon}^{*}\right|^{p+1} d z=\int_{\Omega_{\varepsilon}}\left|w_{\varepsilon}\right|^{p+1} d y \\
& \leq\left(\frac{1}{S_{N}} \int_{\Omega_{\varepsilon}}\left|\Delta w_{\varepsilon}\right|^{2} d y\right)^{(p+1) / 2} \leq C,
\end{aligned}
$$

here we have used the Sobolev inequality for $H^{2} \cap H_{0}^{1}$ functions and (3.6). Let us take $q=p, y=0, \Omega=\Omega_{\varepsilon}^{*}$ in Lemma 4. Thus for $R>0$ in Lemma 4, we obtain

$$
\sup _{B(0, R) \cap \Omega_{\varepsilon}^{*}}\left|w_{\varepsilon}^{*}\right| \leq C\left[\frac{1}{R^{N}} \int_{B(0,2 R) \cap \Omega_{\varepsilon}^{*}}\left|w_{\varepsilon}^{*}\right|^{p+1} d z\right]^{1 /(p+1)} \leq C .
$$
result.

By Lemma 9 and Theorem 7 (2.4), we have the following convergence

Lemma 10. Let $\omega \subset \Omega$ be any neighborhood of $\partial \Omega$ not containing 0 . Then we have

$$
\left\|u_{\varepsilon}\right\|^{2} \frac{\left(u_{\varepsilon}-v_{\varepsilon}\right)}{\left\|u_{\varepsilon}-v_{\varepsilon}\right\|} \rightarrow-2(N-2)(N-4) \sigma_{N} b G(\cdot, 0) \quad \text { in } C^{3}(\omega) .
$$

Proof. We see

$$
\Delta^{2}\left(\left\|u_{\varepsilon}\right\|^{2} \frac{\left(u_{\varepsilon}-v_{\varepsilon}\right)}{\left\|u_{\varepsilon}-v_{\varepsilon}\right\|}\right)=\frac{\left\|u_{\varepsilon}\right\|^{2}}{\left\|u_{\varepsilon}-v_{\varepsilon}\right\|} d_{\varepsilon}(x)\left(u_{\varepsilon}-v_{\varepsilon}\right)=: f_{\varepsilon}(x)
$$

for $x \in \Omega$ with the boundary condition 


$$
\left\|u_{\varepsilon}\right\|^{2} \frac{\left(u_{\varepsilon}-v_{\varepsilon}\right)}{\left\|u_{\varepsilon}-v_{\varepsilon}\right\|}=\Delta\left(\left\|u_{\varepsilon}\right\|^{2} \frac{\left(u_{\varepsilon}-v_{\varepsilon}\right)}{\left\|u_{\varepsilon}-v_{\varepsilon}\right\|}\right)=0
$$

on $\partial \Omega$, where

$$
d_{\varepsilon}(x)=c_{0} p_{\varepsilon} \int_{0}^{1}\left[t u_{\varepsilon}(x)+(1-t) v_{\varepsilon}(x)\right]^{p_{\varepsilon}-1} d t, \quad x \in \Omega .
$$

Note that

$$
\frac{1}{\left\|u_{\varepsilon}\right\|^{p_{\varepsilon}-1}} d_{\varepsilon}\left(\frac{y}{\left\|u_{\varepsilon}\right\|^{\left(p_{\varepsilon}-1\right) / 4}}\right)=c_{\varepsilon}(y), \quad y \in \Omega_{\varepsilon},
$$

see (3.2). Thus

$$
\left|d_{\varepsilon}(x)\right| \leq C \frac{\left\|u_{\varepsilon}\right\|^{-\left(p_{\varepsilon}-1\right)}}{|x|^{(N-4)\left(p_{\varepsilon}-1\right)}}
$$

for any $x \in \Omega, x \neq 0$ by (2.1) and (3.10).

We have by (3.12) and (3.8),

$$
\begin{aligned}
f_{\varepsilon}(x) & =\left\|u_{\varepsilon}\right\|^{2} d_{\varepsilon}(x) w_{\varepsilon}\left(\left\|u_{\varepsilon}\right\|^{\left(p_{\varepsilon}-1\right) / 4} x\right) \\
& \leq C\left\|u_{\varepsilon}\right\|^{2} \frac{\left\|u_{\varepsilon}\right\|^{-\left(p_{\varepsilon}-1\right)}}{|x|^{(N-4)\left(p_{\varepsilon}-1\right)}} \frac{1}{\left(\left\|u_{\varepsilon}\right\|^{\left(p_{\varepsilon}-1\right) / 4}|x|\right)^{N-4}} \\
& \leq C \frac{\left\|u_{\varepsilon}\right\|^{3-p_{\varepsilon}-\left(p_{\varepsilon}-1\right)(N-4) / 4}}{|x|^{(N-4) p_{\varepsilon}}} \rightarrow 0
\end{aligned}
$$

for any $x \neq 0$, since $3-p_{\varepsilon}-\left(p_{\varepsilon}-1\right)(N-4) / 4=-8 /(N-4)+\varepsilon(N / 4)<0$ for $\varepsilon>0$ small.

Also by using (3.10), (3.8), (3.3), (2.1) and the dominated convergence theorem, we obtain

$$
\begin{aligned}
\int_{\Omega} f_{\varepsilon}(x) d x & =\left\|u_{\varepsilon}\right\|^{2-\left(p_{\varepsilon}-1\right) N / 4} \int_{\Omega_{\varepsilon}} d_{\varepsilon}\left(\frac{y}{\left\|u_{\varepsilon}\right\|^{\left(p_{\varepsilon}-1\right) / 4}}\right) w_{\varepsilon}(y) d y \\
& =\left\|u_{\varepsilon}\right\|^{p_{\varepsilon}+1-\left(p_{\varepsilon}-1\right) N / 4} \int_{\Omega_{\varepsilon}} c_{\varepsilon}(y) w_{\varepsilon}(y) d y \\
& \rightarrow c_{0} p \int_{\boldsymbol{R}^{N}} U^{p-1} w d y=c_{0} p b \int_{\boldsymbol{R}^{N}} \frac{1-|y|^{2}}{\left(1+|y|^{2}\right)^{N / 2+3}} d y \\
& =c_{0} p b \sigma_{N}\left(\int_{0}^{\infty} \frac{r^{N-1}}{\left(1+r^{2}\right)^{N / 2+3}} d r-\int_{0}^{\infty} \frac{r^{N+1}}{\left(1+r^{2}\right)^{N / 2+3}} d r\right) \\
& =-2(N-2)(N-4) b \sigma_{N} .
\end{aligned}
$$


Last integrals are computed by the formula

$$
\int_{0}^{\infty} \frac{r^{\alpha}}{\left(1+r^{2}\right)^{\beta}} d r=\frac{\Gamma((\alpha+1) / 2) \Gamma(\beta-(\alpha+1) / 2)}{2 \Gamma(\beta)} .
$$

From these, we confirm that

$$
f_{\varepsilon} \rightarrow-2(N-2)(N-4) \sigma_{N} b \delta_{0}
$$

in the sense of distributions. On the other hand, we can apply the $L^{p}$-theory of [8] to the equation (3.11) with the Navier boundary condition to get

$$
\left\|\Delta\left(\left\|u_{\varepsilon}\right\|^{2} \frac{\left(u_{\varepsilon}-v_{\varepsilon}\right)}{\left\|u_{\varepsilon}-v_{\varepsilon}\right\|}\right)\right\|_{C^{1, \alpha}(\omega)} \leq C(\omega)\left(\left\|f_{\varepsilon}\right\|_{L^{1}(\Omega)}+\left\|f_{\varepsilon}\right\|_{L^{\infty}\left(\omega^{\prime}\right)}\right)
$$

for $\omega \subset \subset \omega^{\prime}$ is a neighborhood of $\partial \Omega$ not containing 0 . Since we have seen that RHS of the above estimate is bounded by a constant independent of $\varepsilon$, Ascoli-Arzelá theorem implies that the function $\Delta\left(\left\|u_{\varepsilon}\right\|^{2}\left(u_{\varepsilon}-v_{\varepsilon}\right) /\left\|u_{\varepsilon}-v_{\varepsilon}\right\|\right)$ converges to some function in $C^{1, \alpha}$-topology. Finally, (3.13) implies that this limit function is $-2(N-2)(N-4) \sigma_{N} b G(x, 0)$.

In the following, we will use Theorem 7 with $x_{\varepsilon}=x_{0}=0$. Recall the Pohozaev identity for $u_{\varepsilon}$ and $v_{\varepsilon}$ ([14] or [17]):

$$
\begin{aligned}
& A_{\varepsilon} \varepsilon \int_{\Omega} u_{\varepsilon}^{p_{\varepsilon}+1} d x=\int_{\partial \Omega}\left(x \cdot \nabla u_{\varepsilon}\right) \frac{\partial \bar{u}_{\varepsilon}}{\partial v} d s_{x}, \\
& A_{\varepsilon} \varepsilon \int_{\Omega} v_{\varepsilon}^{p_{\varepsilon}+1} d x=\int_{\partial \Omega}\left(x \cdot \nabla v_{\varepsilon}\right) \frac{\partial \bar{v}_{\varepsilon}}{\partial v} d s_{x},
\end{aligned}
$$

where

$$
-\Delta u_{\varepsilon}=\bar{u}_{\varepsilon}, \quad-\Delta v_{\varepsilon}=\bar{v}_{\varepsilon}
$$

and

$$
A_{\varepsilon}=\frac{c_{0}(N-4)^{2}}{2(2 N-(N-4) \varepsilon)} .
$$

Subtracting (3.15) from (3.14), and writing as $u_{\varepsilon}^{p_{\varepsilon}+1}-v_{\varepsilon}^{p_{\varepsilon}+1}=h_{\varepsilon}(x)\left(u_{\varepsilon}-v_{\varepsilon}\right)$ where

$$
h_{\varepsilon}(x)=\left(p_{\varepsilon}+1\right) \int_{0}^{1}\left[t u_{\varepsilon}(x)+(1-t) v_{\varepsilon}(x)\right]^{p_{\varepsilon}} d t
$$

we have 


$$
\begin{aligned}
& A_{\varepsilon} \varepsilon \int_{\Omega} h_{\varepsilon}(x)\left(u_{\varepsilon}-v_{\varepsilon}\right) d x \\
& \quad=\int_{\partial \Omega}\left(x \cdot \nabla v_{\varepsilon}\right) \frac{\partial}{\partial v}\left(\bar{u}_{\varepsilon}-\bar{v}_{\varepsilon}\right) d s_{x}+\int_{\partial \Omega}\left(x \cdot\left(\nabla u_{\varepsilon}-\nabla v_{\varepsilon}\right)\right) \frac{\partial}{\partial v} \bar{u}_{\varepsilon} d s_{x} .
\end{aligned}
$$

Let us multiply both sides of (3.16) by $\left\|u_{\varepsilon}\right\|^{3} /\left\|u_{\varepsilon}-v_{\varepsilon}\right\|$. Noting that

$$
\begin{aligned}
& \lim _{\varepsilon \rightarrow 0}\left\|u_{\varepsilon}\right\| u_{\varepsilon}=\lim _{\varepsilon \rightarrow 0}\left\|u_{\varepsilon}\right\| v_{\varepsilon}=2(N-2)(N-4) \sigma_{N} G(\cdot, 0), \\
& \lim _{\varepsilon \rightarrow 0}\left\|u_{\varepsilon}\right\| \bar{u}_{\varepsilon}=\lim _{\varepsilon \rightarrow 0}\left\|u_{\varepsilon}\right\| \bar{v}_{\varepsilon}=2(N-2)(N-4) \sigma_{N}(-\Delta G)(\cdot, 0)
\end{aligned}
$$

in $C^{1}(\omega)$ by (2.3), Lemma 10 and Lemma 3 , we see that

$$
\begin{aligned}
& \frac{\left\|u_{\varepsilon}\right\|^{3}}{\left\|u_{\varepsilon}-v_{\varepsilon}\right\|} \times(\text { RHS of }(3.16)) \\
& =\int_{\partial \Omega}\left(x \cdot \nabla\left(\left\|u_{\varepsilon}\right\| v_{\varepsilon}\right)\right) \frac{\partial}{\partial v}\left(\frac{\left\|u_{\varepsilon}\right\|^{2}}{\left\|u_{\varepsilon}-v_{\varepsilon}\right\|}\left(\bar{u}_{\varepsilon}-\bar{v}_{\varepsilon}\right)\right) d s_{x} \\
& \quad+\int_{\partial \Omega}\left(x \cdot \nabla\left(\frac{\left\|u_{\varepsilon}\right\|^{2}}{\left\|u_{\varepsilon}-v_{\varepsilon}\right\|}\left(u_{\varepsilon}-v_{\varepsilon}\right)\right)\right) \frac{\partial}{\partial v}\left(\left\|u_{\varepsilon}\right\| \bar{u}_{\varepsilon}\right) d s_{x} \\
& \rightarrow-8(N-4)^{2}(N-2)^{2} \sigma_{N}^{2} b \int_{\partial \Omega}(x \cdot \nabla G) \frac{\partial(-\Delta G)}{\partial v}(x, 0) d s_{x} \\
& =-8(N-4)^{3}(N-2)^{2} \sigma_{N}^{2} b R(0) .
\end{aligned}
$$

On the other hand, by using (2.4), (3.8) and the dominated convergence theorem, we have

$$
\begin{aligned}
& \frac{\left\|u_{\varepsilon}\right\|^{3}}{\left\|u_{\varepsilon}-v_{\varepsilon}\right\|} \times(\text { LHS of }(3.16)) \\
& =A_{\varepsilon} \times \varepsilon\left\|u_{\varepsilon}\right\|^{2} \times \int_{\Omega} h_{\varepsilon}(x) \frac{\left\|u_{\varepsilon}\right\|}{\left\|u_{\varepsilon}-v_{\varepsilon}\right\|}\left(u_{\varepsilon}-v_{\varepsilon}\right) d x \\
& =A_{\varepsilon} \times \varepsilon\left\|u_{\varepsilon}\right\|^{2} \times\left(p_{\varepsilon}+1\right)\left\|u_{\varepsilon}\right\|^{1-\left(p_{\varepsilon}-1\right) N / 4+p_{\varepsilon}} \\
& \quad \times \int_{0}^{1} \int_{\Omega_{\varepsilon}}\left[t \frac{1}{\left\|u_{\varepsilon}\right\|} u_{\varepsilon}\left(\frac{y}{\left\|u_{\varepsilon}\right\|^{\left(p_{\varepsilon}-1\right) / 4}}\right)\right. \\
& \left.\quad+(1-t) \frac{1}{\left\|u_{\varepsilon}\right\|} v_{\varepsilon}\left(\frac{y}{\left\|u_{\varepsilon}\right\|^{\left(p_{\varepsilon}-1\right) / 4}}\right)\right]^{p_{\varepsilon}} w_{\varepsilon}(y) d y d t \\
& \rightarrow \frac{c_{0}(N-4)^{2}}{4 N}\left(\lim _{\varepsilon \rightarrow 0} \varepsilon\left\|u_{\varepsilon}\right\|^{2}\right)(p+1) \int_{\boldsymbol{R}^{N}} U^{p} w(y) d y
\end{aligned}
$$




$$
\begin{aligned}
& =C(N) \int_{\boldsymbol{R}^{N}} \frac{1}{\left(1+|y|^{2}\right)^{(N+4) / 2}} \frac{b\left(1-|y|^{2}\right)}{\left(1+|y|^{2}\right)^{(N-2) / 2}} d y \\
& =C(N) b \int_{\boldsymbol{R}^{N}} \frac{1-|y|^{2}}{\left(1+|y|^{2}\right)^{N+1}} d y=0
\end{aligned}
$$

where $C(N)$ is a constant depending only on $N$. Here we have used (2.1) and Theorem 7 (2.5). Hence by (3.17) and (3.18), we have $b=0$.

Step 3. $w \equiv 0$ leads to a contradiction.

By step 1 and step 2, we deduce that the limit function $\lim _{\varepsilon \rightarrow 0} w_{\varepsilon}=w \equiv 0$. Since $\left\|w_{\varepsilon}\right\|=1$, there exists $x_{\varepsilon} \in \Omega_{\varepsilon}$ such that $w_{\varepsilon}\left(x_{\varepsilon}\right)=1$ and $\left|x_{\varepsilon}\right| \rightarrow \infty$ because the above convergence $w_{\varepsilon} \rightarrow w \equiv 0$ is uniformly on compact sets of $\boldsymbol{R}^{N}$. But this is not possible because of Lemma 9 (3.8).

Thus we have proved Theorem 1 .

\section{A nondegeneracy result}

In this section, we will prove Theorem 2. First, we observe that the first eigenvalue $\lambda_{1, \varepsilon}$ of the linearized operator $\mathscr{L}_{\varepsilon}=\Delta^{2}-c_{0} p_{\varepsilon} u_{\varepsilon}^{p_{\varepsilon}-1} I d$ is negative. Indeed, by a variational characterization of $\lambda_{1, \varepsilon}$, we have

$$
\begin{aligned}
\lambda_{1, \varepsilon} & =\inf _{\phi \in H^{2} \cap H_{0}^{1}(\Omega)} \frac{\left(\mathscr{L}_{\varepsilon} \phi, \phi\right)_{L^{2}(\Omega)}}{\int_{\Omega} \phi^{2} d x} \\
& =\inf _{\phi \in H^{2} \cap H_{0}^{1}(\Omega)} \frac{\int_{\Omega}|\Delta \phi|^{2} d x-c_{0} p_{\varepsilon} \int_{\Omega} u_{\varepsilon}^{p_{\varepsilon}-1} \phi^{2} d x}{\int_{\Omega} \phi^{2} d x} \\
& \leq \frac{\int_{\Omega}\left|\Delta u_{\varepsilon}\right|^{2} d x-c_{0} p_{\varepsilon} \int_{\Omega} u_{\varepsilon}^{p_{\varepsilon}+1} d x}{\int_{\Omega} u_{\varepsilon}^{2} d x}=\frac{c_{0}\left(1-p_{\varepsilon}\right) \int_{\Omega} u_{\varepsilon}^{p_{\varepsilon}+1} d x}{\int_{\Omega} u_{\varepsilon}^{2} d x}<0 .
\end{aligned}
$$

Now, the unique solution $u_{\varepsilon}$ to $\left(P_{\varepsilon}\right)$ is obtained by a mountain pass theorem applied to the functional

$$
J_{\varepsilon}(u)=\frac{1}{2} \int_{\Omega}|\Delta u|^{2} d x-\frac{c_{0}}{p_{\varepsilon}+1} \int_{\Omega}|u|^{p_{\varepsilon}+1} d x
$$

defined on $H^{2} \cap H_{0}^{1}(\Omega)$. Thus by Hofer's theorem ([13]), the Morse index of $u_{\varepsilon}$ is at most 1 . Since we see

$$
D^{2} J_{\varepsilon}\left(\phi_{1, \varepsilon}, \phi_{1, \varepsilon}\right)=\int_{\Omega}\left|\Delta \phi_{1, \varepsilon}\right|^{2} d x-c_{0} p_{\varepsilon} \int_{\Omega} u_{\varepsilon}^{p_{\varepsilon}-1} \phi_{1, \varepsilon}^{2} d x=\lambda_{1, \varepsilon} \int_{\Omega} \phi_{1, \varepsilon}^{2} d x<0
$$

for the first eigenfunction $\phi_{1, \varepsilon}$, we must have that the second eigenvalue $\lambda_{2, \varepsilon}$ of $\mathscr{L}_{\varepsilon}$ satisfies $\lambda_{2, \varepsilon} \geq 0$. At this point, we have only to prove that 
Claim: $\lambda_{2, \varepsilon}>0$ for sufficiently small $\varepsilon>0$.

Proof. Suppose the contrary that $\lambda_{2, \varepsilon}=0$ and there exists a solution $w_{\varepsilon}$ to

$$
\begin{cases}\Delta^{2} w_{\varepsilon}=c_{0} p_{\varepsilon} u_{\varepsilon}^{p_{\varepsilon}-1} w_{\varepsilon} & \text { in } \Omega, \\ w_{\varepsilon}=\Delta w_{\varepsilon}=0 & \text { on } \partial \Omega\end{cases}
$$

for $\varepsilon \downarrow 0$. We may assume that $\left\|w_{\varepsilon}\right\|=\left\|u_{\varepsilon}\right\|$ without losing generality. We set

$$
\tilde{w}_{\varepsilon}(y):=\frac{1}{\left\|u_{\varepsilon}\right\|} w_{\varepsilon}\left(\frac{y}{\left\|u_{\varepsilon}\right\|^{\left(p_{\varepsilon}-1\right) / 4}}\right), \quad y \in \Omega_{\varepsilon}=\left\|u_{\varepsilon}\right\|^{\left(p_{\varepsilon}-1\right) / 4} \Omega .
$$

We obtain

$$
\begin{cases}\Delta^{2} \tilde{w}_{\varepsilon}=c_{0} p_{\varepsilon} \tilde{u}_{\varepsilon}^{p_{\varepsilon}-1} \tilde{w}_{\varepsilon} & \text { in } \Omega_{\varepsilon}, \\ \tilde{w}_{\varepsilon}=\Delta \tilde{w}_{\varepsilon}=0 & \text { on } \partial \Omega_{\varepsilon} \\ \left\|\tilde{w}_{\varepsilon}\right\|_{L^{\infty}\left(\Omega_{\varepsilon}\right)}=1 . & \end{cases}
$$

By standard elliptic estimates, $\tilde{w}_{\varepsilon}$ converges to some function $w_{0}$ uniformly on compact sets on $\boldsymbol{R}^{N}$. As in the previous section, we also know $\int_{\Omega_{\varepsilon}}\left|\Delta \tilde{\boldsymbol{w}}_{\varepsilon}\right|^{2} d y \leq$ $C$ and thus $\int_{R^{N}}\left|\Delta w_{0}\right|^{2} d y \leq C$ for some $C>0$. Passing to the limit in (4.2) with noting (2.6), we obtain that $w_{0}$ solves

$$
\left\{\begin{array}{l}
\Delta^{2} w_{0}=c_{0} p U^{p-1} w_{0} \quad \text { in } \boldsymbol{R}^{N} \\
\left\|w_{0}\right\|_{L^{\infty}\left(\boldsymbol{R}^{N}\right)} \leq 1
\end{array}\right.
$$

Thus again by Lemma 8 , we have

$$
w_{0}=\sum_{j=1}^{N} a_{j} \frac{y_{j}}{\left(1+|y|^{2}\right)^{(N-2) / 2}}+b \frac{1-|y|^{2}}{\left(1+|y|^{2}\right)^{(N-2) / 2}}
$$

for some $a_{j}(j=1, \ldots, N), b \in \boldsymbol{R}$.

Now, we recall the following fact, which is a special case of more general result; see Lemma 13 in Appendix.

Lemma 11. Let $\Omega$ be a smooth bounded domain satisfying (H1), (H2)'. Then any solution $w_{\varepsilon}$ to $(4.1)$ is symmetric with respect to the hyperplane $\left\{x_{i}=0\right\},(i=1, \ldots, N)$.

Thanks to lemma 11 , we see $a_{j}=0$ for all $j=1, \ldots, N$ in (4.3), because from the symmetry of the solution $w_{\varepsilon}$ to (4.1), $w_{0}$ also has to be symmetric with respect to the hyperplane $\left\{y_{j}=0\right\}$ for $j=1, \ldots, N$.

Next we will prove that $b=0$ in (4.3). First we show an identity, which is obtained similarly as in [11]. 
Lemma 12. Let $u_{\varepsilon}$ be a solution of $\left(P_{\varepsilon}\right)$ and $w_{\varepsilon}$ be a solution of (4.1). Then we have

$$
\int_{\partial \Omega}\left(\frac{\partial \bar{u}_{\varepsilon}}{\partial v} \frac{\partial w_{\varepsilon}}{\partial v}+\frac{\partial u_{\varepsilon}}{\partial v} \frac{\partial \bar{w}_{\varepsilon}}{\partial v}\right)(x \cdot v) d s_{x}=0
$$

here $\bar{u}_{\varepsilon}=-\Delta u_{\varepsilon}$ and $\bar{w}_{\varepsilon}=-\Delta w_{\varepsilon}$.

Proof. Set $\eta_{\varepsilon}=x \cdot \nabla u_{\varepsilon}$. By direct computation, we have

$$
\begin{gathered}
\Delta \eta_{\varepsilon}=2 \Delta u_{\varepsilon}+x \cdot \nabla\left(\Delta u_{\varepsilon}\right), \\
\Delta^{2} \eta_{\varepsilon}=4 \Delta^{2} u_{\varepsilon}+x \cdot \nabla\left(\Delta^{2} u_{\varepsilon}\right),
\end{gathered}
$$

thus

$$
\Delta^{2} \eta_{\varepsilon}=4 c_{0} u_{\varepsilon}^{p_{\varepsilon}}+c_{0} p_{\varepsilon} u_{\varepsilon}^{p_{\varepsilon}-1} \eta_{\varepsilon} \quad \text { in } \Omega .
$$

Multiplying this equation by $w_{\varepsilon},(4.1)$ by $\eta_{\varepsilon}$, and subtracting, we have

$$
\int_{\Omega}\left(\left(\Delta^{2} \eta_{\varepsilon}\right) w_{\varepsilon}-\left(\Delta^{2} w_{\varepsilon}\right) \eta_{\varepsilon}\right) d x=\int_{\Omega} 4 c_{0} u_{\varepsilon}^{p_{\varepsilon}} w_{\varepsilon} d x
$$

Green's formula implies that

$$
\int_{\Omega} c_{0} u_{\varepsilon}^{p_{\varepsilon}} w_{\varepsilon} d x=\int_{\Omega} \Delta^{2} u_{\varepsilon} \cdot w_{\varepsilon} d x=\int_{\Omega} \Delta^{2} w_{\varepsilon} \cdot u_{\varepsilon} d x=\int_{\Omega} c_{0} p_{\varepsilon} u_{\varepsilon}^{p_{\varepsilon}} w_{\varepsilon} d x
$$

so we have

$$
\int_{\Omega} u_{\varepsilon}^{p_{\varepsilon}} w_{\varepsilon} d x=0
$$

On the other hand, Navier boundary condition implies

$$
\eta_{\varepsilon}=(x \cdot v) \frac{\partial u_{\varepsilon}}{\partial v}, \quad \Delta \eta_{\varepsilon}=x \cdot \nabla\left(\Delta u_{\varepsilon}\right)=(x \cdot v) \frac{\partial\left(\Delta u_{\varepsilon}\right)}{\partial v}
$$

on $\partial \Omega$. Thus we obtain

$$
\begin{aligned}
\int_{\Omega}\left(\left(\Delta^{2} \eta_{\varepsilon}\right) w_{\varepsilon}-\left(\Delta^{2} w_{\varepsilon}\right) \eta_{\varepsilon}\right) d x \\
\quad=\int_{\Omega}\left(\Delta\left(\Delta \eta_{\varepsilon}\right) \cdot w_{\varepsilon}-\Delta \eta_{\varepsilon} \cdot \Delta w_{\varepsilon}\right) d x-\int_{\Omega}\left(\Delta\left(\Delta w_{\varepsilon}\right) \cdot \eta_{\varepsilon}-\Delta w_{\varepsilon} \cdot \Delta \eta_{\varepsilon}\right) d x \\
\quad=\int_{\partial \Omega}\left(\frac{\partial}{\partial v}\left(\Delta \eta_{\varepsilon}\right) w_{\varepsilon}-\left(\Delta \eta_{\varepsilon}\right) \frac{\partial w_{\varepsilon}}{\partial v}\right) d s_{x}-\int_{\partial \Omega}\left(\frac{\partial}{\partial v}\left(\Delta w_{\varepsilon}\right) \eta_{\varepsilon}-\left(\Delta w_{\varepsilon}\right) \frac{\partial \eta_{\varepsilon}}{\partial v}\right) d s_{X}
\end{aligned}
$$




$$
\begin{aligned}
& =\int_{\partial \Omega}\left(\left(-\Delta \eta_{\varepsilon}\right) \frac{\partial w_{\varepsilon}}{\partial v}+\frac{\partial\left(-\Delta w_{\varepsilon}\right)}{\partial v} \eta_{\varepsilon}\right) d s_{x} \\
& =\int_{\partial \Omega}\left(\frac{\partial\left(-\Delta u_{\varepsilon}\right)}{\partial v} \frac{\partial w_{\varepsilon}}{\partial v}+\frac{\partial\left(-\Delta w_{\varepsilon}\right)}{\partial v} \frac{\partial u_{\varepsilon}}{\partial v}\right)(x \cdot v) d s_{x} .
\end{aligned}
$$

Then by (4.5), (4.6) and (4.7), we obtain (4.4).

Using (4.2) and arguing as in Lemma 9, we again have the estimate

$$
\left|\tilde{w}_{\varepsilon}(y)\right| \leq C \frac{1}{|y|^{N-4}} \quad \text { for } y \in \Omega_{\varepsilon} \cap\{|y| \geq \delta\}
$$

for some $\delta>0$. By this estimate, we obtain

$$
\left\|u_{\varepsilon}\right\| w_{\varepsilon} \rightarrow-2(N-2)(N-4) \sigma_{N} b G(\cdot, 0) \quad \text { in } C^{3}(\omega)
$$

where $\omega \subset \Omega$ is any neighborhood of $\partial \Omega$ not containing the origin. The proof of this convergence result is very similar to that of Lemma 10, so we omit it.

Now, we multiply both sides of (4.4) by $\left\|u_{\varepsilon}\right\|^{2}$ to get

$$
\int_{\partial \Omega}\left(\frac{\partial\left(\left\|u_{\varepsilon}\right\| \bar{u}_{\varepsilon}\right)}{\partial v} \frac{\partial\left(\left\|u_{\varepsilon}\right\| w_{\varepsilon}\right)}{\partial v}+\frac{\partial\left(\left\|u_{\varepsilon}\right\| u_{\varepsilon}\right)}{\partial v} \frac{\partial\left(\left\|u_{\varepsilon}\right\| \bar{w}_{\varepsilon}\right)}{\partial v}\right)(x \cdot v) d s_{x}=0 .
$$

By using (2.3), (4.8) and Lemma 3, LHS of (4.9) converges to

$$
\text { LHS } \rightarrow-8(N-2)^{2}(N-4)^{3} \sigma_{N}^{2} b R(0)
$$

as $\varepsilon \rightarrow 0$. Therefore we have $b=0$.

Thus we have proved that $\tilde{w}_{\varepsilon} \rightarrow w_{0} \equiv 0$ uniformly on compact sets of $\boldsymbol{R}^{N}$. Now, the same reason of Step 3 in the previous section is applicable since $\left\|\tilde{w}_{\varepsilon}\right\|_{L^{\infty}\left(\Omega_{\varepsilon}\right)}=1$, therefore we have a desired contradiction. This ends the proof of Theorem 2 .

\section{Appendix}

In this appendix, we show a lemma which is an extension of Theorem 2.1 in [6] to the polyharmonic operator. For this purpose, we recall some definitions.

We say that a $K \times K$ matrix $H=\left(H_{i j}(x)\right)_{1 \leq i, j \leq K}$ with all entries in $C(\bar{\Omega})$ is cooperative if $H_{i j}(x) \geq 0$ for all $i \neq j, 1 \leq i, j \leq K$ and $x \in \bar{\Omega}$. A matrix $H=$ $\left(H_{i j}(x)\right)$ is called fully coupled if for all nonempty sets $I, J \subset\{1, \ldots, K\}$ with $I \cup J=\{1, \ldots, K\}$ and $I \cap J=\phi$, there exist some $i \in I, j \in J$ and $x \in \Omega$ such that $H_{i j}(x) \neq 0$. Let $L$ be a diagonal $K \times K$ matrix of strictly elliptic second order operators and $H=\left(H_{i j}(x)\right), H_{i j} \in C(\bar{\Omega})$. We say $\phi={ }^{t}\left(\phi_{1}, \ldots, \phi_{K}\right) \in$ 
( $\left.W_{\text {loc }}^{2, N}(\Omega) \cap C(\bar{\Omega})\right)^{K}$ is a positive strict supersolution to the system of the second order linear elliptic equations

$$
L \psi=H \psi, \quad \psi={ }^{t}\left(\psi_{1}, \ldots, \psi_{K}\right): \bar{\Omega} \rightarrow \boldsymbol{R}^{K},
$$

if $\phi_{j}(x) \geq 0,((L-H) \phi)_{j}(x) \geq 0$ for all $j=1, \ldots, K$ and $x \in \Omega$, and either $\phi \not \equiv \mathbf{0}$ on $\partial \Omega$ or $(L-H) \phi \not \equiv \mathbf{0}$ in $\Omega$.

In [15] Theorem 1.1, it is proved that if $L$ is as above and $H$ is cooperative and fully coupled, if there is a positive strict supersolution to the system $L \psi=H \psi$ in $\Omega$, and if $\Omega$ satisfies a uniform exterior cone condition, then $\psi=\mathbf{0}$ is the unique solution to

$$
L \psi=H \psi \quad \text { in } \Omega, \quad \psi=\mathbf{0} \quad \text { on } \partial \Omega .
$$

Lemma 13. Let $K \in N$. Let $u$ be a smooth solution of

$$
\begin{cases}(-\Delta)^{K} u=f(u) & \text { in } \Omega, \\ u>0 & \text { in } \Omega, \\ u=\Delta u=\cdots \Delta^{K-1} u=0 & \text { on } \partial \Omega\end{cases}
$$

where $\Omega$ is a smooth bounded domain satisfying ( $\mathrm{H} 1),(\mathrm{H} 2)^{\prime}, f \in C^{1}\left(\boldsymbol{R}_{+}\right)$and $f^{\prime}(u) \geq 0$ for $u>0$. Then any solution of the linearized problem

$$
\begin{cases}(-\Delta)^{K} v=f^{\prime}(u) v & \text { in } \Omega \\ v=\Delta v=\cdots \Delta^{K-1} v=0 & \text { on } \partial \Omega\end{cases}
$$

is also symmetric with respect to the hyperplane $\left\{x_{i}=0\right\},(i=1, \ldots, N)$.

Proof. We rewrite the equation (A.1) to

$$
\begin{cases}-\Delta u_{j}=u_{j+1}=: f_{j}(\mathbf{u}) & \text { in } \Omega,(j=1, \ldots, K-1) \\ -\Delta u_{K}=f\left(u_{1}\right)=: f_{K}(\mathbf{u}) & \text { in } \Omega, \\ u_{j}>0 & \text { in } \Omega,(j=1, \ldots, K) \\ u_{j}=0 & \text { on } \partial \Omega(j=1, \ldots, K)\end{cases}
$$

where $u_{1}=u$ and $\mathbf{u}={ }^{t}\left(u_{1}, u_{2}, \ldots, u_{K}\right)$. Also, setting $v=v_{1}$, we can rewrite the equation (A.2) to

$$
\begin{cases}-\Delta v_{j}=v_{j+1} & \text { in } \Omega, \quad(j=1, \ldots, K-1) \\ -\Delta v_{K}=f^{\prime}\left(u_{1}\right) v_{1} & \text { in } \Omega, \\ v_{j}=0 & \text { on } \partial \Omega(j=1, \ldots, K)\end{cases}
$$

which is, in matrix form,

$$
L \mathbf{v}=H \mathbf{v}, \quad \mathbf{v}={ }^{t}\left(v_{1}, \ldots, v_{K}\right)
$$

for 


$$
L=\left(\begin{array}{ccc}
-\Delta & & 0 \\
& \ddots & \\
0 & & -\Delta
\end{array}\right) \quad \text { and } H=\left(\begin{array}{ccccc}
0 & 1 & 0 & \cdots & 0 \\
\vdots & \ddots & \ddots & \ddots & \vdots \\
\vdots & & \ddots & \ddots & 0 \\
0 & & & \ddots & 1 \\
f^{\prime}\left(u_{1}\right) & 0 & \cdots & \cdots & 0
\end{array}\right)
$$

Note that $H$ is cooperative and fully coupled.

Now, fix $1 \leq i \leq K$. By our assumption of $f$, we easily see

$$
\frac{\partial f_{j}}{\partial u_{i}}(\mathbf{u}) \geq 0, \quad j \neq i, 1 \leq j \leq K .
$$

Therefore we can apply Lemma 4.3 in [16] to get that any solution $\mathbf{u}$ of (A.3) is symmetric with respect to the hyperplanes $\left\{x_{i}=0\right\}$ and $\left(\partial u_{j} / \partial x_{i}\right)>0$ on $\Omega_{i}^{-}=$ $\left\{x \in \Omega \mid x_{i}<0\right\}$ for any $j=1, \ldots, K$. Note also that since the domain $\Omega$ is smooth and symmetric, $\Omega_{i}^{-}$satisfies a uniform exterior cone condition for any $i=1, \ldots, N$.

Set $\phi_{j}=\left(\partial u_{j} / \partial x_{i}\right)$ in $\Omega_{i}^{-}$. From above, we see $\phi_{j}>0$ in $\Omega_{i}^{-}$. Also by elliptic regularity, we see $\phi_{j} \in W_{\text {loc }}^{2, N}\left(\Omega_{i}^{-}\right) \cap C\left(\overline{\Omega_{i}^{-}}\right)$, and $\phi_{j} \not \equiv 0$ on $\partial \Omega \cap \partial \Omega_{i}^{-}$by the Hopf lemma. Moreover, by differentiating the equation of (A.3) with respect to $x_{i}$, we have

$$
\begin{cases}-\Delta\left(\frac{\partial u_{j}}{\partial x_{i}}\right)=\frac{\partial u_{j+1}}{\partial x_{i}} & \text { in } \Omega_{i}^{-},(j=1, \ldots, K-1) \\ -\Delta\left(\frac{\partial u_{K}}{\partial x_{i}}\right)=f^{\prime}\left(u_{1}\right) \frac{\partial u_{1}}{\partial x_{i}} & \text { in } \Omega_{i}^{-},\end{cases}
$$

which is in matrix form, $L \phi=H \phi$ in $\Omega_{i}^{-}$for $\phi={ }^{t}\left(\phi_{1}, \ldots, \phi_{K}\right)$. Therefore, $\phi$ is a positive strict supersolution to the system $L \psi=H \psi$ in $\Omega_{i}^{-}$in the sense described above.

At this point, we can apply Theorem 1.1 of Sweers [15] to get that the system

$$
\begin{cases}L \psi=H \psi & \text { in } \Omega_{i}^{-} \\ \psi=0 & \text { on } \partial \Omega_{i}^{-}\end{cases}
$$

has the only solution $\psi \equiv \mathbf{0}$.

Now, set

$$
\hat{\psi}_{j}(x)=v_{j}\left(x_{1}, \ldots, x_{i}, \ldots, x_{N}\right)-v_{j}\left(x_{1}, \ldots,-x_{i}, \ldots, x_{N}\right), \quad x \in \Omega_{i}^{-}
$$

for $j=1, \ldots, K$, where $\mathbf{v}={ }^{t}\left(v_{1}, \ldots, v_{K}\right)$ is a solution of (A.4). By the symmetry of $\mathbf{u}$, we have

$$
f^{\prime}\left(u_{1}(x)\right)=f^{\prime}\left(u_{1}\left(x_{1}, \ldots,-x_{i}, \ldots, x_{N}\right)\right),
$$


so $\hat{\psi}={ }^{t}\left(\hat{\psi}_{1}, \ldots, \hat{\psi}_{K}\right)$ is a solution of (A.5). Thus $\hat{\psi} \equiv \mathbf{0}$ and $\mathbf{v}$ is symmetric with respect to the hyperplane $\left\{x_{i}=0\right\}$.

Acknowledgement. The authors thank the anonymous referee for carefully reading the manuscript and pointing out an error in an earlier version of this paper. Second author (F. Takahashi) was partially supported by JSPS Grantin-Aid for Scientific Research, No. 17540186 and No. 20540216.

\section{References}

[1] Caristi, G. and Mitidieri, E., Harnack inequality and applications to solutions of biharmonic equations, Oper. Theory Adv. Appl., 168 (2006), 1-26.

[2] Bartsch, T., Weth, T. and Willem, M., A Sobolev inequality with remainder term and critical equations on domains with topology for the polyharmonic operator, Calc. Var. Partial Differential Equations, 18 (2003), 253-268.

[3] Cerqueti, K., A uniqueness result for a semilinear elliptic equation involving the critical Sobolev exponent in symmetric domains, Asymptot. Anal., 21 (1999), 99-115.

[4] Lin, C-S., A classification of solutions of a conformally invariant fourth order equation in $\boldsymbol{R}^{n}$, Comment. Math. Helv., 73 (1998), 206-231.

[5] Chou, K-S. and Geng, D., Asymptotics of positive solutions for a biharmonic equation involving critical exponent, Differential Integral Equations, 13 (2000), 921-940.

[6] Damascelli, L., Grossi, M. and Pacella, F., Qualitative Properties of positive solutions of semilinear elliptic equations in symmetric domains via the maximum principle, Ann. Inst. H. Poincaré Anal. Non Linéaire, 16 (1999), 631-652.

[ 7 ] Djadli, Z., Malchiodi, A. and Ahmedou, M. O., Prescribing a fourth order conformal invariant on the standard sphere. II. Blow up analysis and applications, Ann. Sc. Norm. Super. Pisa Cl. Sci. (5), 1 (2002), 387-434.

[8] Edmunds, D. E., Fortunato, D. and Jannelli, E., Critical exponents, critical dimensions and the biharmonic operator, Arch. Rational Mech. Anal., 112 (1990), 269-289.

[ 9] Felli, V., Existence of conformal metrics on $\boldsymbol{S}^{n}$ with prescribed fourth-order invariant, Adv. Differential Equations, 7 (2002), 47-76.

[10] Gidas, B., Ni, W. M. and Nirenberg, L., Symmetry and related properties via the maximum principle, Comm. Math. Phys., 68 (1979), 209-243.

[11] Grossi, M., A uniqueness result for a semilinear elliptic equation in symmetric domains, Adv. Differential Equations, 5 (2000), 193-212.

[12] Han, Z. C., Asymptotic approach to singular solutions for nonlinear elliptic equations involving critical Sobolev exponent, Ann. Inst. H. Poincaré Anal. Non Linéaire, 8 (1991), 159-174.

[13] Hofer, H., A note on the topological degree at a critical point of mountainpass-type, Proc. Amer. Math. Soc., 90 (1984), 309-315.

[14] Pucci, P. and Serrin, J., A general variational identity, Indiana Univ. Math. J., 35 (1986), 681-703.

[15] Sweers, G., Strong positivity in $C(\bar{\Omega})$ for elliptic systems, Math. Z., 209 (1992), 251-271.

[16] Troy, W. C., Symmetry properties in systems of semilinear elliptic equations, J. Differential Equations, 42 (1981), 400-413. 
[17] van der Vorst, R. C. A. M., Variational identities and applications to differential systems, Arch. Rational Mech. Anal., 116 (1991), 375-398.

[18] Li, Y. Y., Prescribing scalar curvature on $\boldsymbol{S}^{n}$ and related problems, Part I, J. Differential Equations, 120 (1995), 319-410.

\author{
nuna adreso: \\ Tomohiko Sato \\ Computer Centre \\ Gakushuin University \\ 1-5-1 Mejiro, Toshima-ku \\ Tokyo, 171-8588 \\ Japan \\ E-mail: tsato@gakushuin.ac.jp \\ Futoshi Takahashi \\ Department of Mathematics \\ Graduate School of Science \\ Osaka City University \\ Osaka, 558-8585 \\ Japan \\ E-mail: futoshi@sci.osaka-cu.ac.jp
}

(Ricevita la 11-an de marto, 2008)

(Reviziita la 16-an de septembro, 2008) 